\section{ORIGINAL RESEARCH}

\author{
S.P. Ferns \\ C.B.L.M. Majoie \\ M. Sluzewski \\ W.J. van Rooij
}

\title{
Late Adverse Events in Coiled Ruptured Aneurysms with Incomplete Occlusion at 6-Month Angiographic Follow-Up
}

\begin{abstract}
BACKGROUND AND PURPOSE: Patients with coiled ruptured aneurysms with incomplete occlusion at 6 months are not only at risk for rebleed during further follow-up but also for complications of angiographic follow-up and retreatment, and for progressive mass effect by uncontrollable aneurysm growth. We assessed the frequency and outcome of all these possible aneurysm-related events in 124 patients with incompletely occluded aneurysms at 6 months during a follow-up of 419 patient-years.
\end{abstract}

MATERIALS AND METHODS: Between 1994 and 2007, 901 ruptured aneurysms were coiled and 713 (79\%) had 6-month angiographic follow-up, of which 124 were incompletely occluded (17\%). These 124 patients were followed for a mean of 41 months (median, 30 months; range, 1-150 months).

RESULTS: During follow-up, 307 angiograms were obtained without complications. Of 124 aneurysms, 88 were retreated $(71 \%)$. Fifteen aneurysms were retreated more than once. Altogether, 124 additional treatments were performed, and no complications occurred $(0 \% ; 95 \% \mathrm{Cl}, 0.0-3.6 \%)$. Four aneurysms rebled, causing death in 2 patients. Another 4 patients experienced progressive mass effect by growth of the coiled aneurysm, leading to death in 1 . The annual event rate was $1.9 \%$, the annual mortality was $0.7 \%$, and the annual rebleed rate was $1.0 \%$ (8, 3, and 4 in 419 patient-years).

CoNCLUSIONS: In this study of patients with coiled ruptured aneurysms with incomplete occlusion at 6 months, a strategy of imaging follow-up and retreatment when possible leads to a low incidence of serious adverse events. Rebleeding and progressive mass effect of the aneurysm were responsible for these events, not complications from additional treatment or angiographic follow-up.

\begin{abstract}
ABBREVIATIONS: AcomA = anterior communicating artery; AICA $=$ anterior inferior cerebellar artery; Ant = anterior; CARAT = Cerebral Aneurysm Rerupture After Treatment; $\mathrm{Cl}=$ confidence interval; ISAT = International Subarachnoid Aneurysm Trial; $m=$ months; $\mathrm{MCA}=$ middle cerebral artery; PCA = posterior cerebral artery; PcomA = posterior communicating artery; PICA = posterior inferior cerebellar artery; PVO = parent vessel occlusion; SAH = subarachnoid hemorrhage; SCA = superior cerebellar artery
\end{abstract}

E ndovascular treatment with coils has become an established technique for intracranial aneurysms. ${ }^{1}$ A limitation of coiling is that not all aneurysms can be occluded completely at first treatment, and some of the initially adequately occluded aneurysms (with complete or near-complete occlusion) show reopening in time. ${ }^{2-4}$ When aneurysms are adequately occluded at 6-month angiographic follow-up, the risk of late rebleed from the coiled aneurysm during extended follow-up is extremely low. ${ }^{1,5-10}$ On the other hand, patients with aneurysms that are incompletely occluded at 6 months are at risk for late rebleeding. ${ }^{5,6,9}$ In addition, these patients are at risk for other aneurysm-related events: complications from follow-up angiograms and additional treatments and continuous aneurysm growth causing new mass effect. These additional events may also lead to morbidity and mortality at fol-

Received May 12, 2009; accepted after revision July 13.

From the Department of Radiology (S.P.F., C.B.L.M.M.), Academic Medical Center, Amsterdam, the Netherlands; and Department of Radiology (M.S., W.J.v.R.), St. Elisabeth Hospital, Tilburg, the Netherlands.

This work was supported by a grant from the Nuts Ohra Foundation, the Netherlands.

Please address correspondence to Sandra P. Ferns, MD, Department of Radiology, Academic Medical Center Amsterdam, Meibergdreef 9, 1105 AZ Amsterdam, the Netherlands; e-mail: S.P. Ferns@amc.uva.nl

Endicates article with supplemental on-line table

DOI 10.3174/ajnr.A1841 low-up. ${ }^{11,12}$ The magnitude and separate contributions of these factors are not yet elucidated.

The aim of this study was to assess the cumulative incidence of all aneurysm-related adverse events in patients with coiled aneurysms that were incompletely occluded at 6-month angiographic follow-up.

\section{Materials and Methods}

\section{Patient Selection}

Between 1994 and 2007, we prospectively entered 1084 consecutive patients with 1206 coiled intracranial aneurysms into a data base in the St. Elisabeth Hospital in Tilburg, the Netherlands (Fig 1). Nine hundred one aneurysms were ruptured, and 305 aneurysms were unruptured (25.3\%). Of 901 ruptured aneurysms, the mean size was 7.7 $\mathrm{mm}$ (median, $6 \mathrm{~mm}$; range, $2-35 \mathrm{~mm}$ ). Aneurysm location was the anterior circulation in $688(76 \%)$ and the posterior circulation in 213 (24\%) (On-line Table 1). In the 901 ruptured aneurysms, direct postcoiling angiography showed adequate occlusion in 851 (94.5\%) and incomplete occlusion in 50 aneurysms (5.5\%). Angiographic follow-up 6 months after coiling (mean, 6.4 months; median, 6 months) was performed in 713 patients with 713 ruptured aneurysms $(79.1 \%)$. In 188 patients with 188 ruptured aneurysms, no 6-month angiographic follow-up was performed for the following reasons: $111 \mathrm{pa}-$ tients had died ( 80 patients as a consequence of initial SAH, 18 from procedural complications, 6 from unrelated causes, and 7 from early 


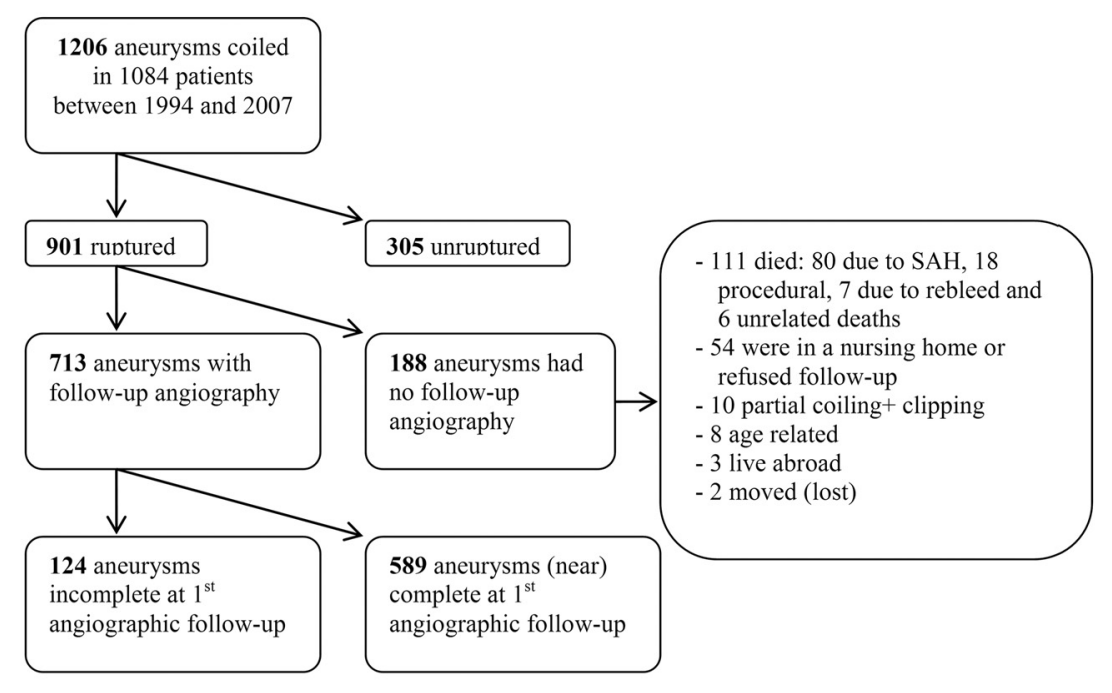

Fig 1. Flow chart showing patient selection.

rebleeding of the coiled aneurysm); 54 patients refused follow-up or were in a nursing home; aneurysms in 10 patients were clipped after incomplete coiling; in 8 patients older than 80 years of age, follow-up angiography was not judged indicated; 3 patients emigrated to another country; and 2 patients moved and could not be traced (Fig 1). Of 713 patients with 713 ruptured aneurysms with 6-month follow-up angiography, 124 aneurysms in 124 patients were incompletely occluded (17.4\%), and these patients form the subjects of this study.

For patients who were not currently being followed up periodically in our center, we first contacted the general practitioner to inquire if the patient was still alive. The medical records of patients who had died during the follow-up period were reviewed to retrieve the exact cause of death. All other patients were contacted by telephone to inquire about late adverse events.

\section{Coiling Procedure}

Coiling of aneurysms was performed with the patient under general anesthesia. In most patients, systemic heparinization was used and heparin was continued intravenously or subcutaneously for 48 hours after the procedure. Coiling was performed with various types of standard platinum coils. The aim of the coiling was to obtain attenuated packing of the aneurysm until no additional coil could be placed. Initial angiographic results of coiling were classified as adequate or incomplete occlusion.

\section{Follow-Up Schedule}

Patients were scheduled for angiographic follow-up at 6 months. Results of angiographic follow-up were classified in the same way as the initial postembolization occlusion. Incomplete occlusion at any point in time was considered an indication for additional therapy, unless clinical or anatomic factors dictated otherwise (unfavorable neck-todome ratio or a very small remnant). Results and consequences of clinical and angiographic follow-up were discussed in a weekly joint meeting with neuroradiologists, neurosurgeons, and neurologists. When appropriate, during the meeting a decision was made as to the need for additional treatment or extended angiographic follow-up. When additional treatment was performed, the result was evaluated in the weekly meeting and 6-month follow-up angiography was scheduled.

\section{Data Analysis}

For aneurysms with incomplete occlusion at 6-month follow-up, the following characteristics were compared with those of aneurysms with adequate occlusion: mean size and proportions of small $(0-5$ $\mathrm{mm})$, medium $(6-10 \mathrm{~mm})$, large $(11-24 \mathrm{~mm})$, and giant $(\geq 25 \mathrm{~mm})$ aneurysms; proportions of aneurysms located in the posterior circulation; and proportion of aneurysms located on the basilar tip. The $\chi^{2}$ test was used for comparison of proportions, and the sample $t$ test, for comparison of means.

The frequency of aneurysm-related adverse events beyond the 6-month follow-up angiography was calculated as a proportion with a $95 \%$ CI. For all adverse events, the incidence rate and mortality per patient-year with $95 \% \mathrm{CI}$ were calculated. The same was done separately for episodes of confirmed rebleeding, for the occurrence of new mass effect, and for complications of follow-up angiography and additional treatments. Survival analysis was used for graphic visualization of the cumulative incidence of late adverse events with time. The follow-up period for survival analysis started at the 6-month follow-up angiography. Reasons for censoring (losses from the sample before the final outcome is observed) were death from causes other than a late adverse event and the end of clinical follow-up. For aneurysms with late adverse events, mean size and aneurysm location were compared with the remaining aneurysms without late events by using a sample $t$ test and the McNemar test.

Number, timing, type, and results of additional treatments were recorded. For incompletely occluded aneurysms that were not retreated, the reason for this decision was noted. All patients with aneurysm-related adverse events were separately described.

\section{Results}

\section{Incompletely Occluded Aneurysms Compared with Adequately Occluded Aneurysms at 6-Month Follow-Up} Of 124 aneurysms with incomplete occlusion at 6-month follow-up, 99 were initially adequately occluded and 25 were incompletely occluded with coils from the onset. Stable incomplete occlusion was present in 13 of 124 aneurysms, and 111 had shown worsening of aneurysm occlusion. Characteristics of incompletely and adequately occluded aneurysms at 6-month follow-up are summarized in On-line Table 1. 


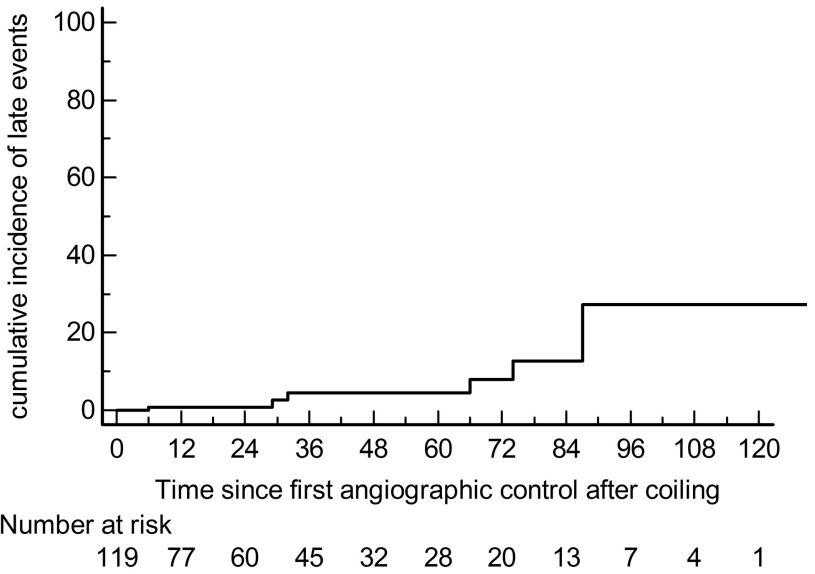

Fig 2. Cumulative incidence of late adverse events in incompletely occluded aneurysms at 6-month follow-up.

The mean size of 124 incompletely occluded aneurysms was $11.4 \mathrm{~mm}$, and of 589 adequately occluded aneurysms, 6.9 $\mathrm{mm}(P<.001)$. The proportion of large- and giant-sized aneurysms was higher in incompletely occluded compared with adequately occluded aneurysms (39\% versus $16 \%$ and $6 \%$ versus $0 \%$, both $P<.001$ ), and the proportion of small-sized aneurysms was higher in adequately occluded aneurysms (45\% versus $19 \%, P<.001)$.

The proportion of basilar tip aneurysms was higher in incompletely occluded compared with adequately occluded aneurysms (23\% versus $13 \%, P=.006$ ), but the proportion of all posterior circulation aneurysms together was not different (31\% versus $23 \%, P=.11$ ).

\section{Cumulative Incidence of Late Adverse Events}

During a mean follow-up of 40.6 months (median, 30 months; range, 1-150 months; 419 patient-years), 124 incompletely occluded aneurysms were at risk for late adverse events. In total, 8 events occurred in 8 patients: Four patients had a rebleed from the coiled aneurysm, and 4 patients developed new symptoms of mass effect. There were no late adverse events from complications of additional treatments or follow-up angiograms. The annual overall aneurysm-related event rate was $1.9 \%$ ( 8 in 419 patient-years; $95 \%$ CI, $0.6-3.2 \%$ ). The annual rate of late rebleeding was $1.0 \%$ (4 in 419 patient-years; $95 \%$ CI, $0.0-1.9 \%)$. The overall annual mortality rate from late adverse events was $0.7 \%$ ( 3 in 419 patient-years; 95\% CI, $0.1-$ $1.5 \%)$. The cumulative proportion of overall aneurysm-related late events and the cumulative proportion of the mortality of late events are graphically displayed in Figs 2 and 3.

The mean size of 8 aneurysms with a late adverse event was larger than the mean size of 116 aneurysms without adverse events (18 versus $11 \mathrm{~mm}, P<.001$ ).

Basilar tip aneurysms were responsible for late adverse events in 4 of 8 patients (50\%). Basilar tip aneurysms were significantly more frequently involved in late adverse events than aneurysms in other locations $(14 \%$ versus $4 \%, P<.001)$.

\section{Additional Treatments and Angiographic Follow-Up} Of 124 incompletely occluded aneurysms at 6-month followup, 88 were additionally treated. Seventy-three were additionally treated with coils, 12 were clipped, and 3 were treated by

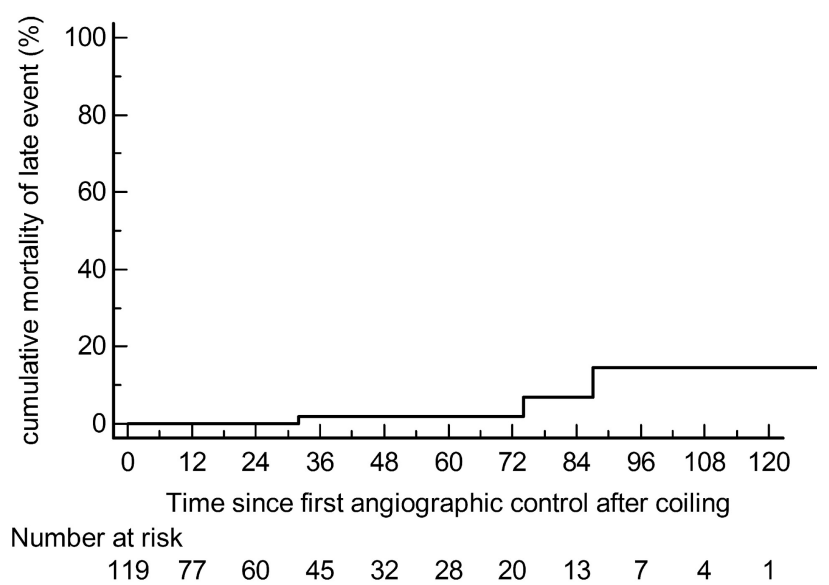

Fig 3. Cumulative mortality from late adverse events in incompletely occluded aneurysms at 6-month follow-up.

parent-vessel occlusion ( 1 after bypass surgery). Of 73 aneurysms that were additionally coiled, 18 aneurysms (25\%) were later coiled for a third time, and 8 of these were eventually coiled 4-8 times (Fig 4). One aneurysm was clipped after the second coiling, and 2 aneurysms were treated by parent-vessel occlusion after second and third coilings. In total, 124 additional treatments were performed (106 were additional coilings), with no complications (0\%; 95\% CI, 0.0-3.6\%). Disregarding angiograms during retreatment, we performed a total of 307 follow-up angiograms in 124 patients (mean, 2.5 per patient; range, 1-8). There were no complications of follow-up angiograms (0\%; 95\% CI, 0.0-1.5\%). Second treatments were performed at a mean of 12.9 months (median, 9; range, 1-72 months) after initial coiling. Thirty-two aneurysms were additionally treated within a month after 6-month angiographic follow-up, and 78 additional treatments were performed within 12 months after 6-month follow-up. Ten additional treatments were performed long after initial coiling, ranging from 21 to 72 months. Reasons for very late additional treatments were the following: availability of new and easy-to-place intracranial stents ${ }^{13}(n=3)$, prolonged follow-up $(n=5)$, proved growth of the aneurysm remnant between the first and second follow-up $(n=1)$, and retreatment in a 78-year-old patient that was not considered necessary until a new mass effect occurred after 72 months.

Thirty-six incompletely occluded aneurysms were not additionally treated for the following reasons: unfavorable geometry of the aneurysm remnant $(n=28)$, advanced patient age $(n=3)$, failed additional coiling $(n=2)$, refused retreatment $(n=1)$, scheduled retreatment $(n=1)$, and serious comorbidity $(n=1$.) The total angiographic follow-up duration of these patients was 1004 years, with a total of 76 angiograms (mean, 2.1 follow-up angiograms per patient; range, 1-4). In all 29 patients with $\geq 2$ follow-up angiograms, the aneurysm remnant remained stable.

\section{Late Rebleeds}

Three patients had a confirmed late rebleed from an incompletely occluded aneurysm. One additional patient likely died as a result of a rebleed, and we included this patient in further analysis (Table). Three of these patients were reported in a previous follow-up study. ${ }^{7}$ The first patient, a 72-year-old 


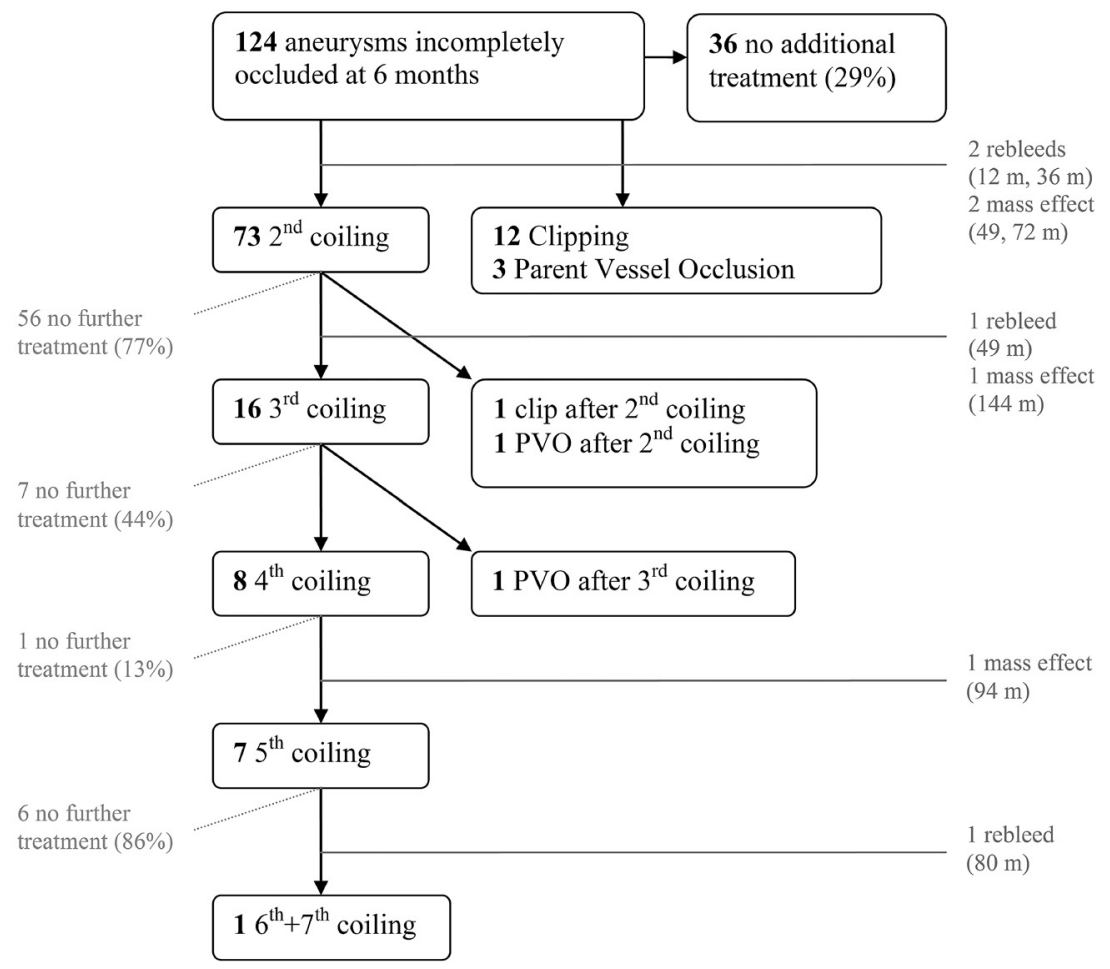

Fig 4. Flow chart of all treatments.

\begin{tabular}{|c|c|c|c|c|c|c|}
\hline \multicolumn{7}{|c|}{ Late adverse events from incompletely occluded aneurysms at 6-month follow-up angiography } \\
\hline Sex, Age (yr) & Aneurysm Size $(\mathrm{mm})$ & Aneurysm Location & Initial Occlusion & Type of Late Event & Timing of Event $(\mathrm{m})$ & Outcome \\
\hline F, 72 & 15 & Basilar tip & Adequate & Rebleed & 12 & Recoil \\
\hline F, 52 & 16 & PcomA & Adequate & Rebleed & 40 & Died \\
\hline M, 63 & 17 & AcomA & Adequate & Rebleed & 36 & Recoil \\
\hline$F, 60$ & 16 & Basilar trunk & Adequate & Rebleed & 80 & Died \\
\hline F, 76 & 3 & PcomA & Incomplete & Mass effect & 72 & Recoil \\
\hline M, 39 & 30 & Basilar tip & Adequate & Mass effect & 144 & Recoil \\
\hline$F, 69$ & 30 & Basilar tip & Adequate & Mass effect & 39 & Died \\
\hline M, 39 & 17 & Basilar tip & Adequate & Mass effect & 94 & Recoil \\
\hline
\end{tabular}

woman with a 15-mm basilar tip aneurysm, presented with a rebleed 12 months after coiling. She had refused 6-month follow-up angiography. Angiography at the time of additional coiling showed incomplete aneurysm occlusion. We assumed that at 6 months, the aneurysm would have been incompletely occluded as well. The aneurysm was additionally coiled with good results.

The second patient was a 52-year-old woman with a 16-mm PcomA aneurysm, which was additionally coiled after 7 months. Follow-up after the second coiling was refused, but eventually, 2 years later, an angiogram showed enormous enlargement and reopening of the aneurysm. At that time, a third coiling was refused. Three months later (40 months after initial coiling), she was readmitted with rebleeding, from which she died 1 day later.

The third patient, a 63-year-old man, had an adequately coiled 17-mm AcomA aneurysm and 2 additional aneurysms on the PcomA and MCA. Seven-month follow-up angiography showed reopening of the AcomA aneurysm with unsuitable geometry for retreatment and unchanged additional aneurysms. At 11 and 30 months, the AcomA remnant was stable. At 36 months, the patient rebled from the AcomA aneurysm and the aneurysm was additionally coiled with good results. In the same session, the additional PcomA aneurysm was coiled with good results. The patient finally died of rupture of the additional MCA aneurysm 48 months after initial coiling of the AcomA aneurysm.

The fourth patient was a 60 -year-old woman with a partially thrombosed 16-mm basilar trunk aneurysm, which was coiled 5 times during 65 months. Follow-up 13 months after the fifth coiling showed reopening, and a sixth coiling was planned. However, she was found dead in her home 4 weeks later. Because this patient had no additional aneurysms and no concomitant other disease, we presumed she had died from a rebleed of the coiled aneurysm.

\section{Late New Mass Effect}

Four patients presented with late new mass effect by growth of the aneurysm, probably caused by dissection or intramural bleeding in an organizing mural thrombus (Table). The first patient, a 76-year-old woman, had a 3-mm PcomA aneurysm, 

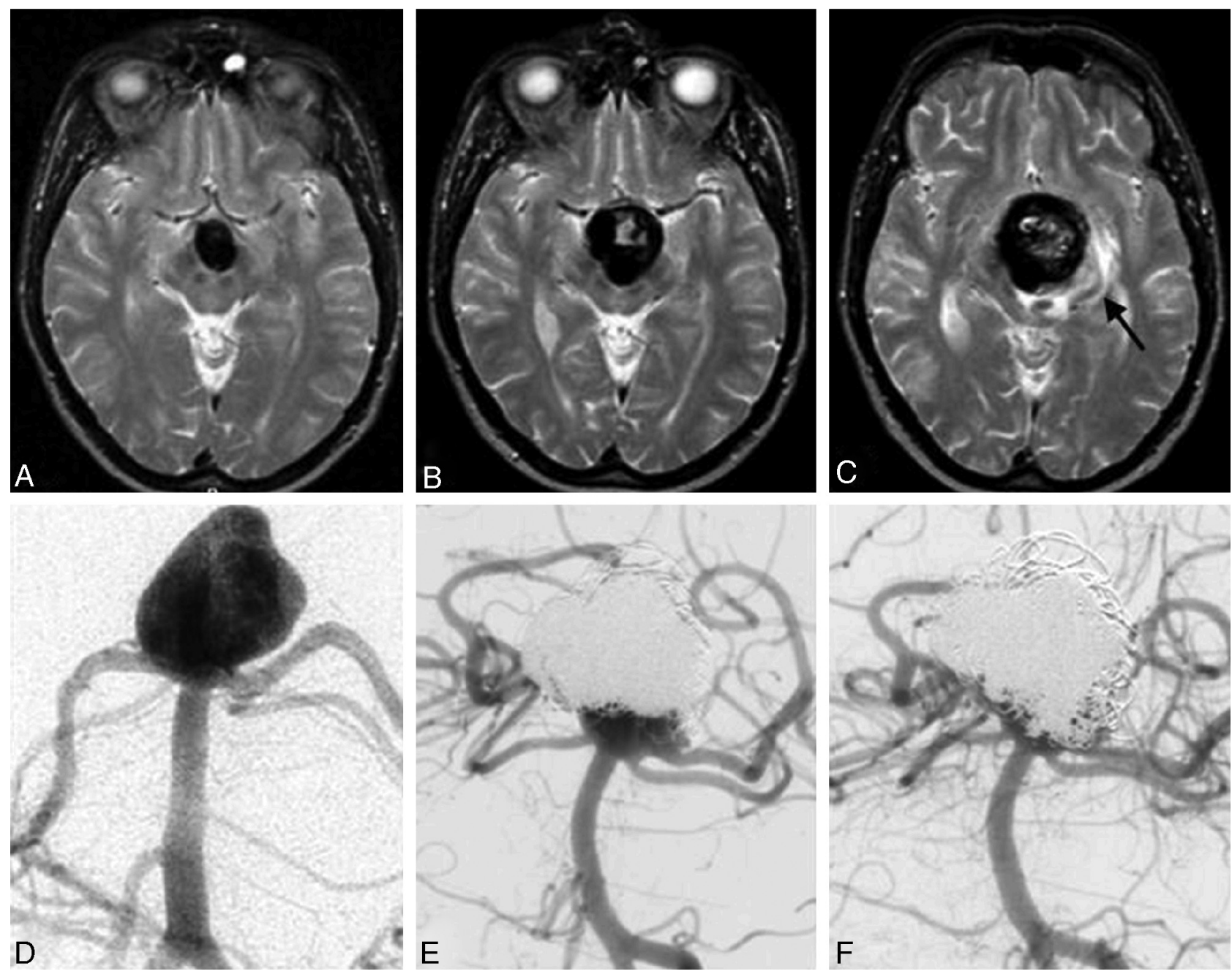

Fig 5. Late mass effect by continuous growth of a large basilar tip aneurysm despite repeated coiling in a 39-year old man. $A-C, M R$ imaging after $2(A), 4(B)$, and $8(C)$ years shows gradual enlargement with brain stem compression and edema (arrow in C). D. Angiogram of the 17-mm basilar tip aneurysm at first presentation after SAH. $E$, Angiogram after 8 years and 4 coiling procedures at the time of presentation with new mass effect (compare with $C$ ). F, Angiogram after the fifth coiling with stent placement shows adequate aneurysm occlusion.

which was incompletely occluded after coiling and remained so at 6-month angiographic follow-up. She was not additionally treated because of advanced age and small aneurysm size. However, she presented with oculomotor palsy 72 months after initial treatment. Angiography showed that the aneurysm had enlarged, and it was additionally coiled. Three months later, the oculomotor palsy was cured.

The second patient, a 39-year-old man, had a 30-mm basilar tip aneurysm, which was initially adequately occluded with coils. At 7-month follow-up, the aneurysm had partially reopened. During 109 months of follow-up, the aneurysm gradually increased in size and was coiled 4 times with good final results. After 144 months, he presented with cognitive decline caused by progressive brain stem compression of the steadily growing basilar tip aneurysm.

The third patient, a 69-year-old woman, had a 30-mm ruptured basilar tip aneurysm, which was initially adequately occluded. After 6 months, the aneurysm had partially reopened and was coiled for a second time. Two years later, she was readmitted with subacute symptoms of brain stem compression. MR imaging showed an enlargement of the aneurysm caused by intramural dissection and brain stem edema. She died several days later.
The fourth patient was a 39-year old man with a $17-\mathrm{mm}$ basilar tip aneurysm, whose aneurysm was coiled 4 times in a 52month period with good final results. At 94 months, he presented with subacute headache and drowsiness caused by dissection and growth of the aneurysm, resulting in brain stem compression with surrounding edema (Fig 5). The aneurysm was coiled for a fifth time, now after stent placement, with good results.

\section{Discussion}

In the subset of patients with coiled ruptured aneurysms with incomplete occlusion at 6 months, a strategy of imaging follow-up and retreatment when possible leads to a low incidence of serious adverse events. Incompletely occluded aneurysms at 6 months were larger and more often located on the basilar tip than their adequately occluded counterparts.

Late adverse events occurred mostly in large and giant basilar aneurysms and were rebleeds or neurologic deficits by mass effect caused by gradual or subacute growth of the coiled aneurysm. Three of 8 late events were fatal.

No complications occurred in 307 follow-up angiograms and in 124 additional treatments in our center. Also in other studies, complications from additional coil procedures are rare, with reported frequencies of $0-2.7 \%{ }^{3,11,14}$ 
This study focused on patients with a coiled ruptured aneurysm that was incompletely occluded at 6 months, $17 \%$ of all coiled ruptured aneurysms with angiographic follow-up in our center. The reason for focusing on this small subgroup of aneurysms is that, almost exclusively, these aneurysms are at risk for recurrent hemorrhage and other adverse events. In aneurysms that are adequately occluded at 6 months, late reopening is rare and late rebleeding from the coiled aneurysm is extremely rare. ${ }^{10,15}$

Previous long-term follow-up studies have reported only on late rebleeds as late adverse events without accounting for other possible aneurysm-related late complications. In a recent follow-up study from ISAT, late rebleeds after coiling were compared with those after clipping. ${ }^{16}$ The authors found 10 rebleeds in 8447 person-years in patients treated with coiling or an annual risk of $0.1 \%$. However, in ISAT, most aneurysms were small and located in the anterior circulation. In these types of aneurysms, we found not a single late recurrent hemorrhage in our study. Also in the CARAT study, the annual risk of late recurrent hemorrhage from the index aneurysm was $0.1 \%$, after the first year. ${ }^{5}$ In our study, concerning the subgroup of aneurysms with incomplete occlusion at 6 months, the annual risk of late rebleeding was a factor 10 times higher than that in ISAT and CARAT. In addition to the late rebleedings, patients experienced, in the same frequency, sometimes devastating symptoms of mass effect from gradually growing aneurysms, especially from large aneurysms located on the basilar tip.

This study has several limitations. Although the data were prospectively collected, the study design was retrospective. The low event rate in combination with the small number and proportion of incompletely occluded aneurysms that were not retreated (36 of 124, 29\%) did not allow an estimation of the number of adverse events that would have been prevented with retreatment. Comparison of late adverse events between retreated and not-retreated incompletely occluded aneurysms in a retrospective study is sensitive to bias. Geometric properties of the aneurysm remnant greatly influence the decision of whether to proceed with additional coiling, and geometric properties may well have been quite different between aneurysms that were additionally treated and those that were not. Early recurrent hemorrhages from the coiled aneurysms were deliberately not included in this study. In many long-term follow-up studies, early recurrent hemorrhage accounts for almost all recurrent hemorrhages. By excluding early recurrent hemorrhages, we tried to focus on long-term outcome only. The strategy of periodic imaging follow-up and retreatment when possible and needed is validated only by the frequency of late adverse events. We have reported on early events in our patient cohort previously. ${ }^{8}$

Our results indicate that the low procedural risks of angiography and additional coilings may justify retreatment of incompletely occluded aneurysms at 6 months, though the effectiveness in preventing adverse events is not clear. Because repeated reopening after retreatment is frequent, regular imaging follow-up after additional treatment is recommended.

In our patient cohort, ruptured posterior circulation aneurysms were over-represented (24\%). A remarkable finding in this cohort is that primary and additional coiling does not always protect against continuous growth of the aneurysm.
This continuous growth is probably stepwise by repeated dissection of the aneurysm wall originating in an aneurysm remnant or hemorrhage from neovascularity in an organizing mural thrombus. This is especially apparent and alarming in large and giant basilar tip aneurysms in which continuous growth results in progressive brain stem compression. In our limited experience, this process can hardly be slowed down or stopped by additional coiling and steroid treatment. Until now, 1 of 4 patients with growing basilar tip aneurysms died from brain stem compression. Short-term prognosis of 2 other patients seems grim. Perhaps, with longer follow-up, this mechanism of continuous aneurysm growth will be responsible for more deaths from late aneurysm-related events than from late recurrent hemorrhage. In our practice, we now make the utmost effort to completely occlude large and giant aneurysms with gradual growth, in an attempt to delay the symptoms of mass effect (Fig 5). Whether this strategy is effective, is not yet clear.

\section{References}

1. Molyneux A, Kerr R, Stratton I, et al. International Subarachnoid Aneurysm Trial (ISAT) of neurosurgical clipping versus endovascular coiling in 2143 patients with ruptured intracranial aneurysms: a randomised trial. Lancet 2002;360:1267-74

2. Byrne JV, Sohn MJ, Molyneux AJ, et al. Five-year experience in using coil embolization for ruptured intracranial aneurysms: outcomes and incidence of late rebleeding. J Neurosurg 1999;90:656-63

3. Henkes H, Fischer S, Liebig T, et al. Repeated endovascular coil occlusion in $\mathbf{3 5 0}$ of $\mathbf{2 7 5 9}$ intracranial aneurysms: safety and effectiveness aspects. Neurosurgery 2006;58:224-32

4. Raymond J, Guilbert F, Weill A, et al. Long-term angiographic recurrences after selective endovascular treatment of aneurysms with detachable coils. Stroke 2003;34:1398-403

5. CARAT investigators. Rates of delayed rebleeding from intracranial aneurysms are low after surgical and endovascular treatment. Stroke 2006;37:143742. Epub 2006 Apr 20

6. Johnston SC, Dowd CF, Higashida RT, et al. Predictors of rehemorrhage after treatment of ruptured intracranial aneurysms: the Cerebral Aneurysm Rerupture After Treatment (CARAT) study. Stroke 2008;39:120-25. Epub 2007 Nov 29

7. Sluzewski M, van Rooij WJ, Beute GN, et al. Late rebleeding of ruptured intracranial aneurysms treated with detachable coils. AJNR Am J Neuroradiol 2005;26:2542-49

8. Sluzewski M, van Rooij WJ. Early rebleeding after coiling of ruptured cerebral aneurysms: incidence, morbidity, and risk factors. AJNR Am J Neuroradiol 2005;26:1739-43

9. Molyneux AJ, Kerr RS, Yu LM, et al. International subarachnoid aneurysm trial (ISAT) of neurosurgical clipping versus endovascular coiling in 2143 patients with ruptured intracranial aneurysms: a randomised comparison of effects on survival, dependency, seizures, rebleeding, subgroups, and aneurysm occlusion. Lancet 2005;366:809-17

10. Schaafsma JD, Sprengers ME, van Rooij WJ, et al. Long-term recurrent subarachnoid hemorrhage after adequate coiling versus clipping of ruptured intracranial aneurysms. Stroke 2009;40:1758-63

11. Renowden SA, Koumellis P, Benes V, et al. Retreatment of previously embolized cerebral aneurysms: the risk of further coil embolization does not negate the advantage of the initial embolization. AJNR Am J Neuroradiol 2008;29:1401-04

12. Slob MJ, Sluzewski M, van Rooij WJ, et al. Additional coiling of previously coiled cerebral aneurysms: clinical and angiographic results. AJNR Am J Neuroradiol 2004;25:1373-76

13. Peluso JP, van Rooij WJ, Sluzewski M, et al. A new self-expandable nitinol stent for the treatment of wide-neck aneurysms: initial clinical experience. AJNR Am J Neuroradiol 2008;29:1405-08

14. Campi A, Ramzi N, Molyneux AJ, et al. Retreatment of ruptured cerebral aneurysms in patients randomized by coiling or clipping in the International Subarachnoid Aneurysm Trial (ISAT). Stroke 2007;38:1538-44

15. Sprengers ME, Schaafsma J, van Rooij WJ, et al. Stability of intracranial aneurysms adequately occluded 6 months after coiling: a 3T MR angiography multicenter long-term follow-up study. AJNR Am J Neuroradiol 2008;29:1768-74

16. Molyneux AJ, Kerr RS, Birks J, et al. Risk of recurrent subarachnoid haemorrhage, death, or dependence and standardised mortality ratios after clipping or coiling of an intracranial aneurysm in the International Subarachnoid Aneurysm Trial (ISAT): long-term follow-up. Lancet Neurol 2009;8:427-33 